\title{
The State of Cardiac Contractile Proteins during the Diastolic Phase
}

\author{
Ichiro Matsubara, M.D., Naoto Yagi, \\ and Masao Endoh, M.D.
}

\begin{abstract}
The molecular events underlying contraction and relaxation of heart muscle were studied by the $\mathrm{X}$-ray diffraction method. In quiescent heart muscle most myosin heads were in the vicinity of the thick filaments. When heart muscle contracted, myosin heads moved to the vicinity of the thin filaments to react with actin. On relaxation of muscle, myosin heads returned to the thick filaments. However, in cyclically contracting heart muscle a significant fraction of myosin heads remained in the vicinity of the thin filaments until the end of the diastolic phase. Therefore, the molecular state during the diastolic phase was considerably different from that in the quiescent state. Paired-pulse stimulation, which enhanced the tension development, increased the number of myosin heads near the thin filaments not only during the systolic phase but also during the diastolic phase. Thus the diastolic molecular state was modified by inotropic intervention. These findings suggest that the diastolic phase should be regarded as a dynamic phase rather than a static phase.
\end{abstract}

$\mathbf{V}$

ARIOUS mechanical experiments have been carried out in order to clarify the time course of molecular events during contraction and relaxation of heart muscle! These experiments involve, for instance, measurements of tension responses to quick stretch and release, or measurements of . the maximum shortening velocity of muscles released during isometric contraction. Analysis of such measurements is usually based on mechanical models which consist of 3 independent components: a contractile component, a series elastic component, and a parallel elastic component. However, it is difficult to relate each component to a separate structural (or molecular) component in muscle? This difficulty has been taken as a serious limitation of such an approach to mole-

Key Words:

$\mathrm{X}$-ray diffraction

Cross-bridge

Paired-pulse stimulation cular events in muscle?

Another approach is to observe directly the structural changes during contraction and relaxation at the molecular level. This can be achieved by using the X-ray diffraction method4 This technique can be regarded as microscopy using X-ray instead of light or electrons. The advantage of using X-rays is that, because of the short wavelength and the penetrating power of X-rays, one can study the molecular events occurring in living muscles.

The main purpose of this paper is to introduce readers to the $\mathrm{X}$-ray diffraction method applied to heart muscle. The data presented will show that the molecular arrangements thus observed during the diastolic phase are different from those in relaxed muscles.

\section{METHODS}

Specimen Preparation

An excised cross-perfused heart was prepared

Department of Pharmacology, University of Tohoku School of Medicine

Supported by Yamada Science Foundation.

Address for correspondence: Ichiro Matsubara, M.D., Department of Pharmacology, University of Tohoku School of Medicine, Seiryo-machi, Sendai 980, Japan 
by the method described by Suga and Sagawa6 The coronary arterial inflow was supplied from the femoral artery of a donor dog and the coronary outflow was returned to the femoral vein of the donor dog. A papillary muscle, attached to the ventricular septum of the cross-perfused heart, was pulled out of the right ventricle through an incision in the free wall? The muscle was held isometric using the method developed by Suga and Numao, 8 and the tension was recorded with a strain-gauge force transducer. The muscle contracted with a regular sinus rhy thm of approximately 115 beats/min. At the beginning of each experiment, the muscle length was adjusted to $\mathrm{L}_{\max }$, where the developed tension was greatest.

In some experiments papillary muscles detached from the right ventricle were used. These muscles were cross-perfused according to the method described by Endoh and Hashimoto ${ }^{9}$; the anterior septal artery nourishing the papillary muscle was cannulated and connected to the carotid artery of a donor dog. Each muscle was attached to an isometric force transducer, and was stimulated through a pair of electrodes placed at the base of the muscle. This type of preparation was mainly used for the timeresolved X-ray diffraction study (see below).

The detached papillary muscle was also used for recording the diffraction pattern in the "quiescent" state 10 When stimulation was interrupted, the muscle showed spontaneous contractions at a frequency of $0.5-2.0$ beats $/ \mathrm{min}$. The muscles in which the frequency was less than 4 beats/min were chosen as the quiescent preparations.

Papillary muscles in rigor were also used!1 Rigor was induced either by soaking the muscle for 2 days in Tyrode solution containing $1 \mathrm{mM}$ iodoacetate, or by extracting the muscle with glycerol for one to two months.

\section{$X$-ray Diffraction}

In optical and electron microscopy, there are 2 stages in obtaining a magnified image of a specimen? The first step is the scattering of light or electrons by the specimen. The second step is the convergence of the scattered light or electrons by lenses to form an image. In the X-ray diffraction method, the first step corresponds to the recording of a diffraction pattern with an X-ray camera; the scattered X-rays give a diffraction pattern due to the regular arrangements of molecules in the specimen. The second stpe is impracticable with X-rays since there is no lens to converge $\mathrm{X}$-rays. Instead, an image is formed through calculations (Fourier synthesis) based on measurements of the diffraction pattern.

The diffraction pattern of the papillary muscle was obtained by using a double-mirror Franks camera! ${ }^{12}$ The X-ray source was a high power rotating anode generator (Rigaku, type FR) operated at $50 \mathrm{kV}$ with a tube current of 70 $\mathrm{mA}$ (nominal focal size $1 \mathrm{~mm} \times 0.1 \mathrm{~mm}$, viewed at an angle of $6^{\circ}$ ). The muscle was mounted vertically in the $\mathrm{X}$-ray camera and the equatorial diffraction pattern was recorded on X-ray film with a specimen-to-film distance of $45 \mathrm{~cm}$. (The equatorial diffraction pattern consists of reflections produced in the plane perpendicular to the muscle axis.)

An electromagnetic shutter was placed between the X-ray source and the muscle. The shutter was coupled with the tension transducer and passed X-rays only in a specified phase of the cardiac cycle (i.e., an stroboscopic exposure). To obtain the systolic pattern, the shutter was opened when the tension exceeded two-thirds of the peak systolic tension and was closed as the tension fell below half of the peak tension?,10 To obtain the diastolic pattern, the shutter was opened and closed while the tension was at the diastolic level. The cardiac cycle was repeated approximately 10,000 times to record one diffraction pattern.

In recording diffraction patterns from quiescent muscles, the electromagnetic shutter was closed at the beginning of each spontaneous contraction and re-opened several seconds after the end of the contraction. An exposure time of approximately $30 \mathrm{~min}$ was required for recording one pattern.

\section{Time-resolved Recording of the Diffraction Pattern}

In some experiments the diffraction pattern was recorded with a position sensitive X-ray detector instead of X-ray film. The combination of such a detector with a digital memory circuit made it possible to follow the changes in the equatorial diffraction pattern on a fast time basis during contraction and relaxation. ${ }^{13}$ The time resolution thus achieved was $32 \mathrm{msec}$.

\section{Formation of an Image}

Each diffraction pattern showed the 1,0 and 1,1 equatorial reflections from the hexagonal array of the myofilaments; these are the Bragg 


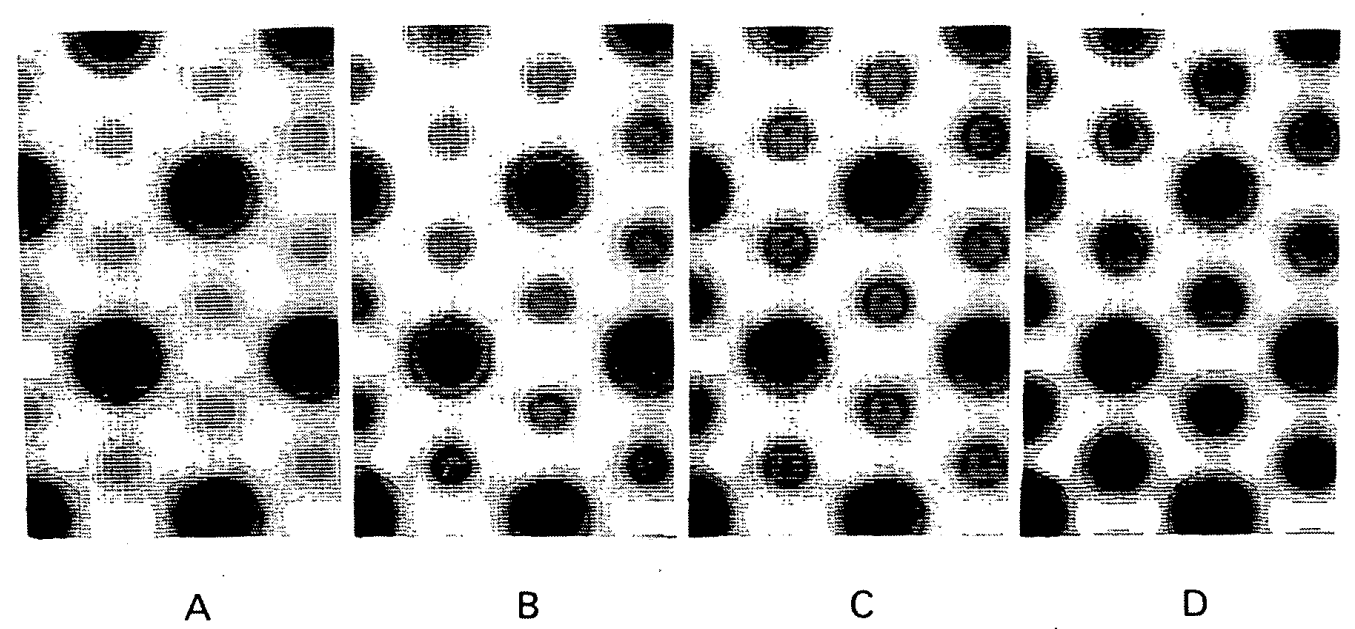

Fig.1. Electron density distribution in the myofilament lattice of heart muscle projected onto the plane perpendicular to the muscle axis. A, quiescent state; $\mathrm{B}$, diastolic state; $\mathrm{C}$, systolic state; D, rigor. (from Matsubara et al. ${ }^{7}$ )

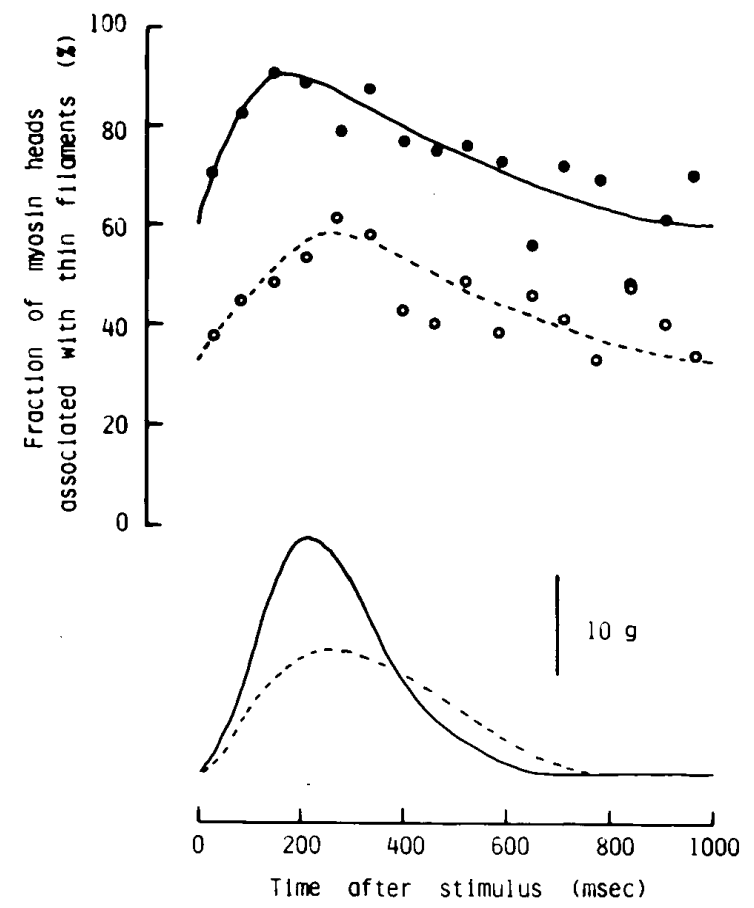

Fig.2. The fraction of myosin heads associated with the thin filaments in the same heart muscle contracting at the same frequency $(1 / \mathrm{min})$ but with different types of stimuli; single pulses (open circles) and paired pulses (filled circles). The tension records (lower panel) are averages of all the contractions needed for recording diffraction patterns with single pulses (dotted line) and paired pulses (solid line). The crosssectional area of the muscle was 0.023 $\mathrm{cm}^{2}$. (from Matsubara et al. ${ }^{13}$ ) reflections arising from the lattice formed by thick and thin filaments. The diffraction pattern recorded on film was densitometered and the integrated intensities of the 2 reflections were measured. When the position sensitive detector was used, the integrated intensities were obtained directly from the intensity distribution curve.

Using these intensities, the electron density distribution in the transverse section of the myofilament lattice was calculated by Fourier synthesis. The calculations were based on the assumption that the phases of the 1,0 and 1,1 reflections are $0^{\circ} 14$

\section{RESULTS}

Figure 1 shows the electron density distribution in the myofilament lattice projected onto the plane perpendicular to the muscle axis under various physiological conditions. The spacial resolution is poor since only the 1,0 and 1,1 intensities were used in the Fourier synthesis. However, the cross-sections of the thick and the thin filaments are clearly visible in each diagram.

The "apparent" diameters of the thick and the thin filaments vary considerably under different physiological conditions. For instance, the apparent diameter of the thin filament is smallest in the quiescent state (A) and largest in rigor (D), while the opposite is true for the thick filament. From these diameters the "apparent" mass of the thick and the thin filaments can be calculated. ${ }^{15}$ Such calculations have shown that the mass of the material associated with the thin 
filaments increases as the muscle shifts from the quiescent state to rigor, and that this is accompanied by a decrease in the mass of the material associated with the thick filaments.

Similar changes in the apparent mass have been observed in skeletal muscle ${ }^{14,15}$ and interpreted to represent the movement of myosin heads between the thick and the thin filaments. The same interpretation is applicable to Fig. 1, when heart muscle shifts from the quiescent state into rigor, myosin heads are transferred from the vicinity of the thick filaments to that of the thin filaments, increasing the apparent mass of the thin filaments and decreasing that of the thick filaments!1

The apparent mass of the thick and the thin filaments during the diastolic (B) and the systolic (C) phases are intermediate between those in the quiescent state and in rigor. This suggests that, compared with the rigor state, a smaller number of myosin heads are associated with the thin filaments during these phases. Calculations have shown that approximately $51-52 \%$ of the total myosin heads are in the vicinity of the thin filaments during the diastolic phase, and $70-71 \%$ during the systolic phase? These calculations were based on the assumption that the mass of the material transferred from the thick to the thin filaments as heart muscle shifts from the quiescent state to rigor represents the total mass of myosin heads.

Thus the above results indicate that the molecular state during the diastolic phase is significantly different from that in the quiescent state, and that more myosin heads are in the vicinity of the thin filaments in the systolic phase than in the diastolic phase. The latter finding strongly supports the idea that the contractile force is produced by myosin heads reacting with actin.

Figure 2 shows the results of the time-resolved experiments in which the effect of paired-pulse stimulation was studied.13 This type of stimulation has been known to increase the peak systolic tension and shortens the time from stimulus to the peak tension. ${ }^{16}$ A muscle was first stimulated once a second with single pulses, the tension development is represented by the dotted line in the lower panel of Fig. 2. The number of myosin heads in the vicinity of the thin filaments (open circles in the upper panel) changed almost in parallel to the tension; such parallelism was confirmed in many other experiments ${ }^{13}$ Then the same muscle was stimulated every second with a pair of pulses to cause a stronger contraction (solid line in the lower panel). The interval between the pair of pulses was set at $270 \mathrm{msec}$ to produce maximal potentiation. The number of myosin heads in the vicinity of the thin filaments at the peak of contraction increased significantly (filled circles in the upper panel), this is likely to be the basis of the inotropic effect. Paired-pulse stimulation also changed the molecular state during the diastolic phase, the number of myosin heads near the thin filaments was increased. This increase was not accompanied by an increase in the diastolic tension.

\section{DISCUSSION}

The X-ray diffraction technique applied to heart muscle has been proved useful in clarifying the molecular events during the cardiac cycle? Concerning the state of the contractile proteins during the diastolic phase, the present study has revealed the following features.

The heads of myosin molecule, which have moved to the vicinity of the thin filaments during contraction, return to the thick filaments during relaxation. The return begins immediately after the peak of contraction and continues even after the tension has fallen to the diastolic level! ${ }^{13}$ When the relaxation is followed by no contraction, the return continues until most myosin heads are found in the vicinity of the thick filaments (i.e., until the molecular state reaches the quiescent state). However, when heart muscle is contracting cyclically, the next contraction starts while a significant proportion of myosin heads still remain in the vicinity of the thin filaments. Therefore, in heart muscle undergoing cyclic contractions the molecular state during the diastolic phase is considerably different from the quiescent state.

The present results have also shown that paired-pulse stimulation modifies the state of the contractile proteins not only during the systolic phase but also during the diastolic phase, with paired-pulses a greater number of myosin heads remain in the vicinity of the thin filaments during the diastolic phase. These myosin heads do not seem to produce a significant force since a change in their number does not affect the diastolic tension. However, their number may be related to the time course and the strength of the subsequent contraction?

The above findings on the molecular state during the diastolic phase suggest that this phase 
should be regarded as a dynamic state rather than a static, inactive state.

\section{Acknowledgment}

We are grateful to Professors K. Hashimoto, N. Taira and $M$. Endo for encouragement.

\section{REFERENCES}

1. BRADY AJ: Active state in cardiac muscle. Physiol Rev 48: 570, 1968

2. JEWELL BR, WILKIE DR: An analysis of the mechanical components in frog's striated muscle. $J$ Physiol 143: 515, 1958

3. SIMMONS RM, JEWELL BR: Mechanics and models of muscular contraction. In Recent Advances in Physiology, ed by LINDEN RJ, Churchill Livingstone, London, 1974

4. MATSUBARA I: X-ray diffraction studies of the heart. Ann Rev Biophys Bioeng 9: 81, 1980

5. TAYLOR CA, LIPSON H: Optical Transforms. G. Bell \& Sons Ltd, London, 1964

6. SUGA H, SAGAWA K: Instantaneous pressurevolume relationships and their ratio in the excised, supported canine left ventricle. Circ Res 35: 117, 1974

7. MATSUBARA I, SUGA H, YAGI N: An X-ray diffraction study of the cross-circulated canine heart. J Physiol 270: 311, 1977

8. SUGA $\mathrm{H}$, NUMAO $\mathrm{Y}$ : In situ papillary muscle preparation and some basic contractile properties. Jap J Physiol 25: 659, 1975

9. ENDOH M, HASHIMOTO K: Frequency-force relationship in the blood-perfused canine papillary muscle. Jap J Physiol 20: 320, 1970

10. MATSUBARA I, KAMIYAMA A, SUGA H: X-ray diffraction study of contracting heart muscle. $J$ Mol Biol 111: 121, 1977

11. MATSUBARA I, MILlMAN BM: X-ray diffraction patterns from mammalian heart muscle. $J \mathrm{Mol}$ Biol 82: 527, 1974

12. FRANKS A: An optically focusing X-ray diffraction camera. Proc Phys Soc B68: 1054, 1955

13. MATSUBARA I, YAGI N, ENDOH M: Movement of myosin heads during a heart beat. Nature 278: 474,1979

14. HUXLEY HE: Structural difference between resting and rigor muscle: Evidence from intensity changes in the low-angle equatorial X-ray diagram. $J$ Mol Biol 37: 507, 1968

15. HASELGROVE JC, HUXLEY HE: X-ray evidence for radial cross-bridge movement and for the sliding filament model in actively contracting skeletal muscle. J Mol Biol 77: 549, 1973

16. BRUTSAERT DL: Studies on the potentiation of contractility of heart papillary muscle by paired stimulation. Arch Intern Physiol Biochim 74: 642, 1966 